$\underline{\text { Preprint typeset in JHEP style - HYPER VERSION }}$

BUHEP-04-05

hep-ph/0405083

\title{
Searching for Composite Neutrinos in the Cosmic Microwave Background
}

\author{
Takemichi Okui \\ Physics Department, Boston University, \\ Boston, MA 02215, USA
}

\begin{abstract}
We analyze signals in the Cosmic Microwave Background (CMB) in theories where the small Dirac neutrino masses arise as a consequence of the compositeness of right-handed neutrinos. In such theories, the right-handed neutrinos are massless "baryons" of a new strong gauge interaction. We find that the results crucially depend on whether or not the new strong sector undergoes chiral symmetry breaking. In the case with chiral symmetry breaking, we find that there are indeed signals in the CMB, but none of them is a direct consequence of neutrino compositeness. In contrast, if the underlying theory does not undergo chiral symmetry breaking, the large scattering cross-section among the composites gives rise to a sizable CMB signal over a wide region of the parameter space, and it can potentially probe whether the neutrino mass spectrum is hierarchical, inverse-hierarchical, or degenerate. We also discuss collider constraints on the compositeness in the context of the CMB signals.
\end{abstract}

Keywords: Neutrinos, Composite Particles, Cosmic Microwave Background. 


\section{Contents}

1. Introduction 1

2. Neutrinos Are Light Because They Are Fat! 3

3. The CMB Signals 5

3.1 The $\Delta N_{\nu}$ Signal

3.2 The $\Delta \ell$ Signal 7

4. Signal Regions In Parameter Space 8

4.1 Case With Chiral Symmetry Breaking 8

4.2 Case Without Chiral Symmetry Breaking

5. Constraints From Terrestrial Experiments 16

6. Conclusions $\quad 20$

\section{Introduction}

While a considerable amount of data has accumulated over the past several years, neutrinos still remain to be one of the most mysterious parts of the standard model of particle physics. Is the neutrino mass spectrum hierarchical, inverse hierarchical, or degenerate? Are neutrino masses Dirac or Majorana? What is the underlying mechanism that generates these incredibly tiny masses - at least six orders of magnitude smaller than the electron mass - and large mixing angles?

Recently, signals in the Cosmic Microwave Background (CMB) that can probe these questions are proposed by Z. Chacko, L. Hall, T.O. and S. Oliver [1]. They investigate signals in theories where small neutrino masses arise from flavor symmetry spontaneously broken at a very low energy scale. The CMB signals arise because such a theory necessarily introduces new particles interacting with neutrinos at sufficiently low energies to generate small neutrino masses. Consequently, properties of the neutrino fluid during the acoustic oscillation at $T \approx \mathrm{eV}$ can be significantly modified, which in turn leaves signals in the CMB. For example, an increase in the energy density 
of the neutrino fluid will appear in CMB observation as a deviation of the effective number of neutrino species from the standard value, namely, three. A change in the scattering property of the neutrino fluid can also affect the CMB; while in the standard cosmology neutrinos are assumed to be free-streaming during the generation of the $\mathrm{CMB}$ at $T \approx \mathrm{eV}$, scattering of neutrinos with the new particles can prevent neutrinos from free-streaming. This effect will appear in the CMB in a very dramatic way - all the acoustic peaks of the CMB spectrum will shift uniformly by the amount $\Delta \ell \approx 8$ for each non-free-streaming neutrino species.

It should be clear, however, that the existence of these signals is qualitatively quite generic to any theory where small neutrino masses are generated at sufficiently low energies, because whatever sector that generates the neutrino masses must involve new particles and new interactions with neutrinos, and if the associated energy scale is sufficiently low, it can affect the CMB spectrum.

In fact, for Dirac neutrinos, another mechanism for naturally generating small masses is known; neutrino masses are tiny because the right-handed neutrinos are composite [2]. One theoretical aspect where this mechanism is clearly more attractive than generating small neutrino masses from low-energy flavor symmetry breaking is that there is no issue of stabilizing a very low VEV required for obtaining small neutrino masses. In a flavor symmetry breaking scenario we need to add some extra ingredient, such as supersymmetry, into the theory to ensure the stability, but as far as the CMB signals are concerned, those extra complications are completely irrelevant. In sharp contrast, the stability of the scale in a composite neutrino scenario is automatically guaranteed thanks to dimensional transmutation. While experimental bounds on the compositeness of the charged fermions have been pushed higher and higher in energy, it is quite interesting that there is large unexplored room for the compositeness of neutrinos, which can be exploited to explain mysteries like small neutrino masses, and which can be tested by looking at the sky.

In this paper we explore questions like: how large are the CMB signals for the composite neutrinos? Can we tell from the CMB signals whether or not the strong sector that produces the composite right-handed neutrinos breaks chiral symmetry dynamically? Can we distinguish a composite-neutrino scenario from a flavor-symmetrybreaking scenario? In section 2, we will review the idea of composite neutrinos introduced in Ref. [2]. In section 3, we will review the CMB signals proposed in Ref. [1], emphasizing the model-independent features relevant for us. Section $\$$ is the main part of the paper in which we will analyze the CMB signals for two qualitatively different scenarios, one with chiral symmetry breaking and the other without. We will show that a very low compositeness scale is necessary for the CMB signals to be generated. In section 5 we will discuss constraints from terrestrial experiments on such a low 
compositeness scale. We will conclude in section 6. In the appendix, we present two concrete models that produce exactly three massless fermionic composites with and without chiral symmetry breaking. Although our discussions of the CMB signals are completely model-independent, it is quite satisfactory to have actual, concrete models that do what we want.

\section{Neutrinos Are Light Because They Are Fat!}

In this section we will briefly review how compositeness can naturally explain tiny neutrino masses [2]. We exclusively deal with the Dirac type neutrinos. ${ }^{1}$ First, note that there is absolutely no difficulty in describing a small Dirac neutrino mass; we can just write down a Yukawa coupling, $\ell \nu_{R} h$, with an appropriately small coefficient. (Here $\ell$ and $h$ are the standard-model lepton and Higgs doublets, respectively, while $\nu_{R}$ is the right-handed neutrino.) However, since neutrino oscillation data [3-6] suggest that the heaviest neutrino mass is about $0.05 \mathrm{eV}$ in the case of a hierarchical or inversehierarchical mass spectrum, we find that even the largest coefficient of this Yukawa coupling is about $3 \times 10^{-13}$. Although there are no theoretical (nor experimental) problems with this small coupling, especially given the fact that the electron Yukawa coupling is $\mathcal{O}\left(10^{-6}\right)$, it is small enough that it is worth attempting to explain the smallness. The idea of composite (or fat) right-handed neutrinos explains quite elegantly why Dirac neutrinos can be so light.

Suppose that there is a new sector with a new asymptotically-free gauge interaction which confines at a scale $\Lambda$, under which all the standard-model fields are neutral. We call this new color force nu-color. Also suppose that the nu-color sector only communicates with the neutrino sector of the standard model, via some irrelevant operators. (No relevant or marginal operators are capable of connecting the two sectors.) More explicitly, suppose that, at some energy scale $M \gg \Lambda$, the following operator is generated:

$$
\mathcal{L}=\cdots+\frac{1}{M^{3(n-1) / 2}} \ell h \underbrace{q q \cdots q}_{n \text { "quarks" }}
$$

where a $q$ schematically refers to a "quark" charged under the nu-color gauge group. (In general there are different kinds of $q$ 's, namely, not necessarily all the "quarks"

\footnotetext{
${ }^{1}$ One way to ensure the Dirac nature of neutrinos is to impose lepton-number (or $B-L$ ) conservation. Another way is to break lepton-number (or $B-L$ ) at an extremely high scale; for example, a Planck-suppressed operator $\ell \ell h h / M_{\mathrm{P} \ell}$, gives an utterly negligible contribution to neutrino masses, so the Dirac-type contributions from the compositeness as described in this section will completely dominate.
} 
are in the same representation of the nu-color group. But since this sort of details are unimportant here, we just collectively call them q.) Since the $q$ 's are fermions, $n$ must be an odd integer.

Now, at the scale $\Lambda$, nu-color dynamics confines and produces a bunch of "baryons", "mesons", "glueballs", etc. Suppose that the confinement produces three massless "baryons" of the form corresponding to the combination $q q \cdots q$ in (2.1), whose masslessness is ensured by some unbroken chiral symmetry of the nu-color dynamics. ${ }^{2} \mathrm{We}$ identify these three massless "baryons" as the three right-handed neutrinos. Then, beneath $\Lambda$, in terms of the canonically-normalized right-handed neutrino fields, $\nu_{R} \approx$ $q q \cdots q / \Lambda^{3(n-1) / 2}$, the operator (2.1) becomes

$$
\mathcal{L}=\cdots+\left(\frac{\Lambda}{M}\right)^{3(n-1) / 2} \ell \nu_{R} h
$$

If we assume for simplicity that the original operator (2.1) has an $\mathcal{O}(1)$ coefficient for the heaviest neutrino in the case of a hierarchical or inverse-hierarchical spectrum, then we obtain the following relation:

$$
\left(\frac{\Lambda}{M}\right)^{3(n-1) / 2} \approx \frac{m_{\nu_{3}}}{v},
$$

where $m_{\nu_{3}} \approx 0.05 \mathrm{eV}$, and $v$ is the electroweak VEV, $174 \mathrm{GeV}$. Because of this power-law relation, a small hierarchy in $\Lambda / M$ can reproduce the large hierarchy in $m_{\nu_{3}} / v$.

Let us study some numerics about $M$ and $\Lambda$. For example, in the "minimal" case of $n=3$, we have

$$
\Lambda \approx 0.7 \times 10^{-4} M \quad \text { if } \nu_{R} \propto q q q \quad(n=3)
$$

while for $n=5$ we have

$$
\Lambda \approx 0.8 \times 10^{-2} M \quad \text { if } \nu_{R} \propto q q q q q \quad(n=5) .
$$

Even though $10^{-4}$ may still be regarded as a small number, it is an "acceptable" small number because the electron Yukawa coupling, for example, is $\mathcal{O}\left(10^{-6}\right)$. This is a significant improvement compared to the original hierarchy, $10^{-13}$.

Note that for the purpose of getting small neutrino masses, only the ratio of $\Lambda$ to $M$ matters. However, in this paper we are interested in lowering the entire scale down

\footnotetext{
${ }^{2}$ For explicit examples of such gauge theories, see Appendix. The examples in the appendix are all 4D non-supersymmetric asymptotically-free gauge theories. A different, interesting framework to realize composite neutrinos is to use the AdS/CFT duality, where the right-handed neutrinos are composites of the CFT [7].
} 
so that there are some effects on the CMB. Then, what is the lower bound on $M$ ? Clearly, we are safe if $M \gtrsim v$. This actually turns out to be the case, as we will see in section 4.1, if the nu-color dynamics undergoes chiral symmetry breaking. On the other hand, if $M$ is lower than $v$, it is not immediately clear whether this is excluded or not, because the answer depends on how the operator (2.1) is generated. We will come back to this point later in detail after we analyze the case without chiral symmetry breaking, where we will see that $\Lambda$ must be indeed very low to generate the CMB signals.

\section{The CMB Signals}

As discussed in [1], there are two qualitatively different signals that can probe how neutrino masses are generated at low energy. Here we will briefly review these signals, emphasizing model-independent, essential features of the signals.

The most important event that must happen before anything else is thermalization among left-handed neutrinos, right-handed neutrinos and other particles from the nucolor sector. Obviously, to generate any signal in the CMB this thermalization must occur before the temperature of the universe becomes $\mathcal{O}(\mathrm{eV})$, since otherwise the evolution of the universe would be completely standard all the way down to the era of the acoustic oscillation. However, the thermalization should not take place too early either, because we know that Big-Bang Nucleosynthesis (BBN) works quite well in the standard cosmology, which in particular constrains the number of relativistic degrees of freedom (converted into the effective number of neutrino species) during the BBN era to be $2.4 \pm 0.8$ at $2 \sigma$ level [8]. ${ }^{3}$ This bound arises because the $\mathrm{D}$ and ${ }^{4} \mathrm{He}$ abundances are very sensitive to the expansion rate of the universe which is proportional to the squareroot of the number of relativistic degrees of freedom. Therefore, we have room only for the standard three left-handed neutrinos, and it is only after BBN can we bring the particles from the nu-color sector into thermal equilibrium with left-handed neutrinos. Following [1], we call this thermalization event neutrino-recoupling, meaning that the left-handed neutrinos experience thermalization once again after they decoupled from the photon-baryon-electron plasma.

\subsection{The $\Delta N_{\nu}$ Signal}

This signal utilize the fact that the CMB spectrum is sensitive to the energy density of relativistic degrees of freedom when the temperature of the universe is $\mathcal{O}(\mathrm{eV})$. If the energy density of relativistic degrees of freedom is increased, the equality of matter

\footnotetext{
${ }^{3}$ Note that this is a constraint from the BBN data alone (i.e. no CMB or large scale structure data are used), which is appropriate for our purpose because we are modifying cosmology in the later era.
} 
and radiation occurs later, so the time window between the equality and the photondecoupling decreases. Consequently, the sound-wave of the photon-baryon plasma has less time to travel, so the size of the sound-horizon at the time of the photon-decoupling shrinks. Since the location of the first CMB peak is the ratio of the present horizon to the sound-horizon then (modulo effects of curvature), we see that increasing the radiation energy density moves the first peak to the right (i.e. higher multipole $\ell$ ). Furthermore, since the gravitational potential due to dark matter has less time to grow before the photon-decoupling, the heights of the potential hills that photons must climb up become lower, so the CMB photons red-shifts less. Hence, the CMB spectrum becomes more blue, i.e., the first $\mathrm{CMB}$ peak not only moves to the right but also becomes higher. Therefore, the CMB spectrum is very sensitive to the radiation energy density at $T \approx \mathrm{eV}[9]$. It is customary in cosmology to express the extra (i.e. other than photons) relativistic degrees of freedom in terms of the effective number of neutrino species. The current bounds from WMAP alone is $0.9 \lesssim N_{\nu} \lesssim 8.3$ at the $2 \sigma$ level [8], while adding late time data such as large scale structure tightens the bound slightly to $1.9 \lesssim N_{\nu} \lesssim 7.0$ [10], which still allows room for nonstandard $N_{\nu}$.

Now we are ready to discuss how $N_{\nu}$ can be different from 3 in our scenario. To illustrate the idea, imagine that in addition to our three composite $\nu_{R}$ 's, the nu-color sector contains another light particle, $\phi$, which couples to neutrinos schematically as $g \nu_{L} \nu_{R} \phi$. (In the flavor symmetry breaking scenarios discussed in Ref. [1], $\phi$ is a pseudoGoldstone boson from the flavor symmetry breaking.) Let us begin the story from the moment right after BBN. Because of the BBN constraint on $N_{\nu}$ we discussed earlier, we are initially allowed to have only $\nu_{L}$ 's in the system. Now, the interaction $g \nu_{L} \nu_{R} \phi$ will bring $\nu_{L}, \nu_{R}$ and $\phi$ into equilibrium before $T$ becomes $\mathcal{O}(\mathrm{eV})$ if $g$ is sufficiently large and the rate of the process $\nu_{L} \bar{\nu}_{L} \rightarrow \phi \phi^{*}$ decreases less rapidly than the expansion rate does. Note that neutrino-recoupling requires $\phi$ to be relativistic, since a scattering process involving non-relativistic $\phi$ in the final state would have a highly suppressed phase space. So after neutrino-recoupling we are left with a gas of relativistic $\nu_{L}, \nu_{R}$ and $\phi$.

Now, if $m_{\phi} \lesssim \mathrm{eV}$, we will just observe $N_{\nu}=3$ in the CMB, because in this case the gas will stay relativistic all the way the down to the matter-radiation equality, and by energy conservation the total energy density of the $\nu_{L^{-}} \nu_{R^{-}} \phi$ gas will be the same as that of the pure $\nu_{L}$ gas in the standard cosmology as if nothing had occurred. Since the CMB is sensitive only to the total energy density and not to the composition of the gas, there will be no observable effect in this case.

On the other hand, if $m_{\phi} \gtrsim \mathrm{eV}$, the $\phi$ particles go non-relativistic before the matter-radiation equality. Recall that a similar situation occurs to electrons when the temperature drops below the electron mass. In that case, electron-positron pairs 
annihilate into photons, heating up the photon temperature by the famous $(11 / 4)^{\frac{1}{3}}$ factor. Exactly the same thing happens in our case as well; pairs of $\phi$ 's annihilate into neutrinos, raising the neutrino temperature. This will appear in the CMB as $N_{\nu}>3$. Clearly, the strength of this signal, or the amount of deviation of $N_{\nu}$ from 3 , depends on how many neutrinos had recoupled to $\phi$ in the first place. For example, in the scenarios discussed in Ref. [1], the coupling $g$ is proportional to $m_{\nu}$, so a heavier neutrino is more likely to recouple than a lighter one, and this is why measuring $N_{\nu}$ from the CMB can tell us how many neutrino species are heavy, by which we can distinguish the three different possible mass spectra of neutrinos. As we will see, an analogous situation occurs in our scenario as well.

\subsection{The $\Delta \ell$ Signal}

This signal is based on an effect of neutrinos on the CMB spectrum which is of completely different nature from the previous one. In the standard cosmology, neutrinos are assumed to be non-interacting and relativistic when $T \approx \mathrm{eV}$. This assumption has a significant consequence as follows. Suppose that we know what the CMB spectrum looks like without taking into account the free-streaming of neutrinos, and then consider how this spectrum gets modified by putting the free-streaming back. While the photon-baryon plasma undergoes acoustic oscillations in the gravitational potential created by dark matter, the neutrinos just free-stream with the speed of light from over-dense regions to under-dense regions. The difference between the speed of this neutrino flow and the speed of sound-waves in the plasma causes extra phase shift in the acoustic oscillation. Bashinsky and Seljak [11] has derived an analytic expression for how the neutrino free-streaming affects the locations of CMB peaks. A striking feature of their result is that the neutrino free-streaming shifts the locations of all the peaks by an equal amount! For our purpose, their result can be cast conveniently as [1]:

$$
\Delta \ell=-57\left(\frac{0.23 N_{\nu}^{F S}}{1+0.23 N_{\nu}}\right)\left(\frac{\delta \ell_{\text {peak }}}{300}\right)
$$

where $N_{\nu}^{F S}$ is the (effective) number of neutrino species which are free-streaming at $T \approx \mathrm{eV}$, while $N_{\nu}$ is the grand total (effective) number of neutrino species during the same era. $\delta \ell_{\text {peak }}$ is the spacing between two successive peaks, which is $\approx 300$. Note that the amount of shift is independent of which peak we are looking at, although one should keep in mind that for the first few peaks it is difficult to isolate this effect from larger peak shifts due to other causes. For higher multipole $\ell$, this signal is very clean and unique.

We have already explained in section 3.1 how $N_{\nu}$ can be different from 3 in our scenario. What about $N_{\nu}^{F S}$ ? How can it be different from $N_{\nu}$ ? One possibility is that 
$N_{\nu}^{F S}$ is less than $N_{\nu}$ due to scattering of neutrinos with $\phi$ 's. If the scattering occurs

sufficiently frequently, it will prevent the neutrinos from free-streaming. ${ }^{4}$ In flavor symmetry breaking scenarios in Ref. [1], this signal is useful for probing the neutrino mass spectrum because $g$ is proportional to $m_{\nu}$ so that a heavier neutrino is less likely to free-stream.

In our case, however, there is an additional process which must be considered. Since our $\nu_{R}$ is a composite object, they can scatter with each other without involving extra particles like $\phi$ or any other long-range forces. In other words, at energies below the compositeness scale $\Lambda$, the Lagrangian contains the operator $\bar{\nu}_{R} \bar{\nu}_{R} \nu_{R} \nu_{R}$ with a large coefficient, thanks to the strong nu-color interaction. This operator leads to important consequences which have no analogs in the flavor symmetry breaking scenarios.

\section{Signal Regions In Parameter Space}

There are two completely different cases in our scenario which must be separately considered. In both cases, the nu-color sector is designed such that confinement produces three massless "baryons" which we identify as three right-handed neutrinos, and their masslessness is guaranteed by low energy chiral symmetry which survives through confinement. Now, the two cases are distinguished by comparing the high energy and low energy flavor symmetries. In the first case, the low energy symmetry is smaller than the high energy symmetry - that is, the nu-color sector undergoes chiral symmetry breaking, producing massless "pions". We take into account a more general possibility that these "pions" may be pseudo-Goldstone bosons, treating their masses to be freeparameters. Therefore, the low energy spectrum of a theory of this type contains these naturally light scalars as well as the three massless right-handed neutrinos.

In the second case, confinement occurs without any chiral symmetry breaking, i.e., the original flavor symmetry of the high energy theory is completely preserved even after confinement. Therefore, in this case, there are no light particles beneath $\Lambda$ other than the three massless right-handed neutrinos.

\subsection{Case With Chiral Symmetry Breaking}

In this case, we have naturally light scalars, "pions", which have interactions with neutrinos at low energies. These interactions may be able to induce neutrino-recoupling.

\footnotetext{
${ }^{4}$ One may think that the possibility of such scattering is disfavored by the analyses in Refs $[12,13]$. However, the crucial difference between their model and ours is the occurrence of neutrino recoupling. Because of recoupling, neutrinos and $\phi$ 's share energy that was originally carried by neutrinos, while in their model neutrinos and $\phi$ 's are already thermalized with the rest of the universe prior to the decoupling of neutrinos.
} 
One may suspect that the results in this section should be identical to those of the Dirac neutrino case discussed in [1] where they have pseudo-Goldstone bosons from flavor symmetry breaking instead of the "pions" - the only difference seems our flavor symmetry is broken by strong dynamics instead of by the VEV of a scalar field. However, that is not the only difference - as we mentioned earlier, the scattering property of the neutrino fluid can be very different because of the compositeness of $\nu_{R}$. So we will focus on this point in this section.

After electroweak symmetry breaking and nu-color confinement, the leading interaction is contained in

$$
\mathcal{L}_{i n t} \approx v\left(\frac{\Lambda}{M}\right)^{3(n-1) / 2} \nu_{L} \nu_{R} \mathrm{e}^{i \pi / f}+\text { h.c. }
$$

where $\pi$ collectively denotes the "pions", and $v$ is the electroweak VEV, and $f \approx \Lambda / 4 \pi$. As we discussed in Sec.2, the ratio $(\Lambda / M)^{3(n-1) / 2}$ is fixed to be $3 \times 10^{-13}$ to get the right neutrino mass. Therefore, (4.1) can be rewritten more nicely as

$$
\mathcal{L}_{i n t} \approx g \nu_{L} \nu_{R} f \mathrm{e}^{i \pi / f}+\text { h.c. }
$$

where

$$
g \equiv \frac{m_{\nu_{3}}}{f}
$$

with $m_{\nu_{3}} \approx 0.05 \mathrm{eV}$.

First, consider a process, $\nu_{L} \nu_{R} \rightarrow \pi \pi$. One might think that this process is impossible because initially there should not be any $\nu_{R}$ 's available in the universe because of the BBN constraint. However, it is very hard to imagine that the number density of $\nu_{R}$ 's is absolutely zero; after all, the post-inflation reheating may well create "quarks" and "gluons" of the nu-color sector as well. So it is more natural to expect that there are some amount of $\nu_{R}$ 's. In fact, the BBN bound allows $N_{\nu}=3.2$ at the $2 \sigma$ level, so as long as the energy density of the $\nu_{R}$ 's is less than a several percent of that of the $\nu_{L}$ 's, there is no conflict with BBN. There are several ways by which the $\nu_{R}$ energy density becomes suppressed. For example, since the nu-color sector is neutral under the standard-model gauge group, the latent heat from the electroweak and/or QCD phase transitions, if any, is transfered only to the standard-model sector. In any case, to account for this possible $\nu_{R}$ suppression, we introduce $r \equiv n_{\nu_{R}} / n_{\nu_{L}}$. The BBN constraint then tells us that if the temperatures of the $\nu_{L}$ and the $\nu_{R}$ are the same, $r$ should be less than 0.1 or so, but actually $r$ can even be $\mathcal{O}(1)$ if the $\nu_{R}$ temperature is less than about a half of the $\nu_{L}$ temperature, which is perfectly fine because the 
two sectors are not in thermal contact before neutrino-recoupling. So, $r$ may be $\mathcal{O}(1)$, $\mathcal{O}(0.1)$, or smaller, but it begins to look very unnatural as $r$ becomes too small.

Now, in order for the process, $\nu_{L} \nu_{R} \rightarrow \pi \pi$, to induce neutrino-recoupling, the pions must be relativistic, since otherwise the cross-section would be highly suppressed by a tiny phase space. However, even in the relativistic limit, the cross-section for this process is not given by $\sim g^{4} / T^{2}$, due to the derivatively-coupled nature of $\pi$; it actually goes as $g^{4} / m_{\nu_{3}}^{2}$. Thus, the rate is given by

$$
\Gamma_{\nu_{L} \nu_{R} \rightarrow \pi \pi} \approx \frac{r g^{4} T^{3}}{16 \pi m_{\nu_{3}}^{2}}
$$

Unfortunately, this rate dies faster than the expansion rate as $T$ drops, so this process cannot recouple neutrinos. Instead, this process is more important at higher temperatures. Of course, it does not become indefinitely more important as $T$ increases, because the above formula assumes $T<\Lambda$. The easiest way to understand the qualitative behavior of the cross-section for $T>\Lambda$ is to look at the similar process in a theory where chiral symmetry is spontaneously broken by a VEV of a strongly-self-coupled scalar. In that case, the "Higgs" mode with mass of $\mathcal{O}(4 \pi \times \mathrm{VEV})$ comes in to the process, and as a result the behavior of the cross-section changes from $\propto 1 / m_{\nu}^{2}$ to $\propto 1 / T^{2}$ for energies larger than $\mathcal{O}(4 \pi \times \mathrm{VEV})$. Similarly, in our case, some resonances come in to the process at $E \approx 4 \pi f \approx \Lambda$ and changes the the cross-section from $\propto 1 / m_{\nu}^{2}$ to $\propto 1 / T^{2}$. Therefore, the rate is actually maximal at $T \approx \Lambda$, compared to the expansion rate.

Similarly, consider another process, $\nu_{L} \nu_{R} \rightarrow \pi \pi \pi$. The rate of this process is given by

$$
\Gamma_{\nu_{L} \nu_{R} \rightarrow \pi \pi \pi} \approx \frac{T^{2}}{16 \pi^{2} f^{2}} \Gamma_{\nu_{L} \nu_{R} \rightarrow \pi \pi}
$$

where the $16 \pi^{2}$ accounts for the 3 -body phase space. Therefore, although this process is subdominant to the previous process at low temperatures, it becomes as important when $T$ reaches near $4 \pi f \approx \Lambda$. This is in fact expected because the theory becomes strongly-coupled at this scale.

Now, if these two processes are occurring at temperatures near $\Lambda$, then all of $\nu_{L}$, $\nu_{R}$ and $\pi$ are brought into equilibrium without chemical potentials. This will lead to a value of $N_{\nu}$ that is too large at the BBN era, so we must avoid it. Hence, we evaluate the rate (4.4) at $T \approx \Lambda$, and demand that it be smaller than the expansion rate:

$$
\frac{r g^{4} \Lambda^{3}}{16 \pi m_{\nu_{3}}^{2}} \lesssim \frac{\Lambda^{2}}{M_{p l}} \quad \Longrightarrow \quad \Lambda \gtrsim\left(16 \pi^{3} r m_{\nu_{3}}^{2} M_{p l}\right)^{\frac{1}{3}} \approx r^{\frac{1}{3}} \mathrm{GeV}
$$

where we have used (4.3). 
On the other hand, in order for the $\nu_{R}$ compositeness to play any role in modifying the CMB spectrum, the rate of the $\nu_{R} \nu_{R} \rightarrow \nu_{R} \nu_{R}$ must be appreciable at $T \approx \mathrm{eV}$, because this is the leading process that can probe the compositeness. At energies beneath $\Lambda$, the compositeness of the $\nu_{R}$ yields the operator

$$
\mathcal{L}_{\text {int }} \approx\left(\frac{4 \pi}{\Lambda}\right)^{2} \bar{\nu}_{R} \bar{\nu}_{R} \nu_{R} \nu_{R}
$$

where we have estimated the coefficient using Naive Dimensional Analysis (NDA) [1417]. Thus, the rate of the $\nu_{R} \nu_{R}$ scattering is given by

$$
\Gamma_{\nu_{R} \nu_{R} \rightarrow \nu_{R} \nu_{R}} \approx \frac{(4 \pi)^{4} T^{5}}{16 \pi \Lambda^{4}}
$$

and demanding that this rate be larger than the expansion rate at $T \approx \mathrm{eV}$ gives rise to

$$
\Lambda<4 \pi\left(\frac{\mathrm{eV}^{3} M_{p l}}{16 \pi}\right)^{\frac{1}{4}} \approx 10 \mathrm{MeV}
$$

Note that this becomes consistent with the BBN bound (4.6) only if $r<10^{-6}$ ! Unless there is a good reason why $r$ can be made so tiny, this is quite implausible.

Therefore, we conclude that if the underlying nu-color sector has chiral symmetry breaking, BBN requires the confinement scale $\Lambda$ to be very high as in (4.6), and in fact it is so high that no sign of the compositeness can be seen at low energy scales relevant for the $\mathrm{CMB}$ (i.e., $T \approx \mathrm{eV}$ ), and consequently the CMB signals are indistinguishable from those in the Dirac neutrino case analyzed in Ref. [1] where small neutrino masses arise from low-energy spontaneous flavor-symmetry breaking by the VEV of a (weaklycoupled) scalar. The reader should look at Ref. [1] for details, but in short the signals in their case arise from the process, $\nu_{L} \nu_{R} \rightarrow G$, where $G$ is a pseudo-Goldstone boson from the flavor-symmetry breaking. This occurs with the rate

$$
\Gamma_{\nu_{L} \nu_{R} \rightarrow G} \approx \frac{r g^{2} m_{G}^{2}}{16 \pi T}
$$

where $m_{G}$ is the mass of the $G$. This reaction clearly can induce recoupling.

It is unfortunate that we cannot distinguish these two different underlying mechanisms for generating small neutrino masses, but a positive way of viewing this is that a nu-color sector with chiral symmetry breaking offers a very natural, simple way to stabilize the very low symmetry-breaking scale appearing in such theories, alternative to the supersymmetric stabilization discussed in Ref. [1]. 


\subsection{Case Without Chiral Symmetry Breaking}

In this case, the three $\nu_{R}$ 's are the only massless particles, and all other "hadrons" have masses of $\mathcal{O}(\Lambda)$. This means that if $\Lambda>\mathrm{MeV}$, those particles are too heavy to participate in neutrino-recoupling, because recoupling must occur at a temperature below $\mathcal{O}(\mathrm{MeV})$ to avoid having too many thermalized relativistic degrees of freedom during BBN. One may wonder if a 4 -fermion operator, $\bar{\ell} \bar{\nu}_{R} \ell \nu_{R}$, can induce neutrino recoupling. The answer is no, because the natural size of the coefficient of this operator is $\mathcal{O}\left(10^{-25}\right)$ because of the compositeness of the $\nu_{R}$, and this is too small to do anything.

What about the operator (4.7)? Can it induce recoupling? Let us consider a process, $\nu_{L} \nu_{L} \rightarrow \bar{\nu}_{R} \bar{\nu}_{R}$, where we put two mass-insertions in the initial state to flip the helicity of each $\nu_{L}$. Note that processes with only one mass insertion such as $\nu_{L} \nu_{R} \rightarrow \nu_{R} \bar{\nu}_{R}$ are forbidden by angular momentum conservation. Hence, the process, $\nu_{L} \nu_{L} \rightarrow \bar{\nu}_{R} \bar{\nu}_{R}$, (and the "bar" of this process) is the leading process that can couple $\nu_{L}$ and $\nu_{R}$ via the operator (4.7). The rate of this process is given by

$$
\Gamma_{\nu_{L} \nu_{L} \rightarrow \nu_{R} \nu_{R}} \approx \frac{(4 \pi)^{4}}{16 \pi \Lambda^{4}} \frac{m_{\nu}^{4}}{T^{4}} T^{5} .
$$

Since this rate dies slower than the expansion rate, it can induce recoupling. Comparing this with $T^{2} / M_{p l}$, we obtain the recoupling temperature:

$$
T_{r e c}^{\nu_{L} \nu_{L} \rightarrow \nu_{R} \nu_{R}} \approx \frac{16 \pi^{3} M_{p l} m_{\nu}^{4}}{\Lambda^{4}} \approx 10\left(\frac{\mathrm{MeV}}{\Lambda}\right)^{4}\left(\frac{m_{\nu}}{0.05 \mathrm{eV}}\right)^{4} \mathrm{eV} .
$$

$\mathrm{BBN}$ requires $T_{\text {rec }}<\mathrm{MeV}$, leading to

$$
\frac{\Lambda}{100 \mathrm{keV}} \gtrsim \frac{m_{\nu}}{0.05 \mathrm{eV}} \text {. }
$$

If the neutrino masses are not degenerate, this bound must be applied for the heaviest one. On the other hand, generating a CMB signal needs recoupling to occur for $T>\mathrm{eV}$, leading to

$$
\frac{\Lambda}{\mathrm{MeV}} \lesssim \frac{m_{\nu}}{0.05 \mathrm{eV}}
$$

Here, we should recall that the $0.05 \mathrm{eV}$ is the mass of the heaviest neutrino if the mass spectrum is hierarchical or inverse hierarchical. In these cases, the recoupling condition (4.14) implies $\Lambda<\mathrm{MeV}$, and in particular this invalidates the BBN bound (4.19) because $\mathrm{BBN}$ occurs at $T \approx \mathrm{MeV}$ which is now higher than $\Lambda$, so the use of the operator (4.7) is not justified. On the other hand, if the mass spectrum is degenerate, $m_{\nu}$ can be larger than $0.05 \mathrm{eV}$, so (4.14) may allow $\Lambda>\mathrm{MeV}$ in which case the bound 
(4.13) applies. However, even in the the degenerate case, $m_{\nu}$ is not expected to be much larger than $0.5 \mathrm{eV} .{ }^{5}$ In other words, $\Lambda$ is not expected in any case to be larger than a several $\mathrm{MeV}{ }^{6}$

So, let us take $\Lambda<\mathrm{MeV}$ and see where it leads to. It seems, then, that there are other routes for neutrino-recoupling and danger of messing up BBN, because now "hadrons" from the nu-color confinement are light enough that they may participate in recoupling and $\mathrm{BBN}$. Let us examine this point. First, if $T<\Lambda$, then we can integrate out all the nu-color composites except the $\nu_{R}$ 's which are massless; we have already analyzed this case above. Therefore, consider a case, $\Lambda<T<M$. In this range, recoupling should occur via the following interaction that is obtained from (2.1) with $\langle h\rangle=v$ :

$$
\mathcal{L}_{i n t} \approx \frac{v}{M^{3(n-1) / 2}} \nu_{L} q q \cdots q
$$

where the $q$ 's here are now unconfined "quarks". However, even for the minimal case of $n=3$, this is a highly irrelevant operator and the rate for a process like $\nu_{L} \bar{\nu}_{L} \rightarrow q q \bar{q} \bar{q}$, for instance, dies away too quickly, so neutrinos cannot recouple. Of course it is even worse for larger $n$. Therefore, we conclude that the recoupling temperature should be higher than $M$. It should be emphasized here that if a process involves an irrelevant coupling, it cannot lead to recoupling, because it requires too many factors of $T$ in the numerator to compensate a power of the mass scale appearing in the denominator. The only way to kill a large power of $T$ in the numerator is to put mass insertions, $m_{\nu}^{2} / T^{2}$, but for $T>\Lambda$ there exist no such mass terms. Therefore, in order to have recoupling, we need a "UV theory" which generates (2.1) without involving any irrelevant operators.

It turns out, however, that we can extract quite a bit of information without knowing exactly what the UV theory is. First of all, the UV theory should involve not only q's but also some additional, "messenger" fields, collectively called $X$, which communicate the standard-model sector with the nu-color sector; interactions of $X$ with $q$ and $\nu_{L}$ should be designed such that at energies beneath the $X$ mass, integrating out $X$ will yield the operator (2.1) (and most likely many other operators of much higher dimension). Secondly, as we argued above, interactions among $X, q$ and $\nu_{L}$ cannot

\footnotetext{
${ }^{5} \mathrm{~A}$ limit on the sum of neutrino masses from large-scale structure is about $0.5-2 \mathrm{eV}$ [18-20]. However, all of these bounds assume that neutrinos free-stream, which is not necessarily true in our scenario. A more direct bound, $m_{\nu}<2.2 \mathrm{eV}$, has been obtained from tritium $\beta$-decay experiments [21-23]. Note that this bound should apply to each of the three mass eigenstates, since neutrino oscillation data imply $\delta m^{2} \ll \mathrm{eV}^{2}$ and large mixing angles [24].

${ }^{6}$ Such a low value of $\Lambda$ and correspondingly a low value of $M$ might have constraints and signals in collider experiments. We will discuss those in some detail in section 5 . In this section we will focus on constraints and signals in cosmological and astrophysical circumstances.
} 
involve irrelevant operators in order to have recoupling. For simplicity, we assume that all relevant operators such as the $X$ mass just involve a single mass scale $m_{X}$.

Now, consider a diagram where we have one $\nu_{L}$ and $n q$ 's in the external states while the internal state involves only $X$ 's. This becomes the operator (2.1) at energies below $m_{X}$. Here we see that the scale $M$ in (2.1) should be identified as

$$
\frac{v}{M^{3(n-1) / 2}} \approx \frac{\lambda}{m_{X}^{(3 n-5) / 2}},
$$

where $\lambda$ is the product of all the coupling constants appearing in the diagram. Also, the relation (2.3) allows us to rewrite this in terms of $\Lambda$ instead of $M$ :

$$
\frac{m_{\nu_{3}}}{\Lambda^{3(n-1) / 2}} \approx \frac{\lambda}{m_{X}^{(3 n-5) / 2}} .
$$

One thing we have to worry about here is whether or not the addition of $X$ messes up the confinement dynamics. To answer this question, note that in order to get neutrino masses, the operator (2.1) should be already there when the " $q q \cdots q$ " confines into $\nu_{R}$. This order cannot be reversed. Therefore, we must impose

$$
m_{X} \gg \Lambda
$$

so that we can integrate out $X$ to get (2.1) before we reach the confinement scale $\Lambda$. This also guarantees that the confinement dynamics is not affected by the addition of $X$. Combining (4.17) and (4.18), we obtain a very simple bound:

$$
\lambda \gg \frac{m_{\nu_{3}}}{\Lambda} \Longrightarrow \frac{\lambda}{10^{-7}} \gg \frac{\mathrm{MeV}}{\Lambda} \text {. }
$$

Now, note that this same diagram can also cause the process, $q \nu_{L} \rightarrow(n-1) \bar{q}$, to occur. Once this happens, then various reactions like $q q \leftrightarrow q q, q q \leftrightarrow X X$, etc. start occurring very rapidly and everything becomes thermalized without chemical potentials, which is bad for BBN. This second step occurs really quickly because these reactions can be mediated by exchanging nu-color gluons, leading to the rate of $\mathcal{O}(T / 16 \pi)$ or larger, which is much faster than the rate of the initial triggering process, $q \nu_{L} \rightarrow(n-1) \bar{q}$. Of course, we also need $T / 16 \pi \gg \Lambda$ so that that they do not confine before thermalization occurs, but since we are analyzing here the case where $T>m_{X} \gg \Lambda, T / 16 \pi$ is bigger than $\Lambda$ by assumption. Therefore, we have to make sure that the initial triggering reaction, $q \nu_{L} \rightarrow(n-1) \bar{q}$, does not happen before BBN. If none of the virtual $X$ 's in this process is a vector or a derivatively-coupled scalar (otherwise recoupling cannot occur), then the rate is simply given by dimensional analysis as

$$
\Gamma_{q \nu_{L} \rightarrow(n-1) q} \approx \frac{r \lambda^{2} T}{16 \pi\left(16 \pi^{2}\right)^{n-3}},
$$


where the factor of $\left(16 \pi^{2}\right)^{n-3}$ accounts for the $(n-1)$-body phase space, while $r$ takes into account the effective fraction of the number density of $q$ 's with respect to that of $\nu_{L}$, analogous to the $r$ in section 4.1. Demanding this rate to be less than the expansion rate at $T \approx \mathrm{MeV}$ gives

$$
\lambda \lesssim r^{-1 / 2}(4 \pi)^{n-2} \times 10^{-11}
$$

Note that this is inconsistent with (4.19) and $\Lambda<\mathrm{MeV}$ unless

$$
r \ll(4 \pi)^{2 n-4} \times 10^{-8} .
$$

For $n=3,5$ this restricts $r$ as $r \ll 10^{-6}, 10^{-2}$, respectively, while $n=7$ gives no restriction.

However, if we suppress the $q \nu_{L} \rightarrow(n-1) \bar{q}$ process by, say, taking $n=7$, then there will be other processes dominating the physics. For example, consider a process, $q \nu_{L} \rightarrow X$. Since the "UV theory" necessarily includes a term like $\nu_{L} q X$ in order for the standard-model and nu-color sectors to be connected via non-irrelevant interactions, this process inevitably exists. Then, the rate of this process is given by

$$
\Gamma_{q \nu_{L} \rightarrow X} \approx \frac{r \lambda^{\prime 2} \tilde{m}_{X}}{16 \pi} \frac{\tilde{m}_{X}}{T}
$$

where $\lambda^{\prime}$ is the product of all the coupling constants appearing in the amplitude, while $\tilde{m}_{X}$ is the effective "temperature-corrected" mass of the $X$, i.e., $\tilde{m}_{X}^{2}=m_{X}^{2}+a^{2} T^{2}$ with some constant $a$. Since $X$ is strongly interacting via the nu-color interaction, $a$ is expected to be $\mathcal{O}(1)$. So, remembering the assumption $T>m_{X}$, we approximate $\tilde{m}_{X}$ as $\tilde{m}_{X} \approx a T$. Then, the process $q \nu_{L} \rightarrow X$ will lead to recoupling at the temperature

$$
T_{r e c}^{q \nu_{L} \rightarrow X} \approx \frac{r \lambda^{\prime 2} a^{2} M_{p l}}{16 \pi}
$$

Once some amount of $X$ 's is produced from this process, various reactions like $q X \leftrightarrow$ $q X, X \leftrightarrow q q$, etc. will begin rapidly via the nu-color gauge interaction and immediately thermalize all of $\nu_{L}, q$ and $X \mathrm{~s}$ with no chemical potentials. Therefore, we have to make sure that the initial triggering process, $q \nu_{L} \rightarrow X$, occurs after BBN. Demanding that the recoupling temperature is less than $\mathrm{MeV}$, we obtain

$$
r \lambda^{\prime 2} \lesssim 10^{-19}
$$

where we have ignored $a$ since it is $\mathcal{O}(1)$. Since our very motivation to consider composite neutrinos is to avoid the very tiny Yukawa couplings for the Dirac neutrinos, we do not want to reintroduce a small Yukawa coupling here. So, if we assume that $\lambda^{\prime}$ is 
no smaller than the electron Yukawa coupling, $r$ must be smaller than $10^{-7}$ ! Unless there is a good reason why $r$ could be made so small, this is quite implausible.

Therefore, we conclude that in any reasonable scenario, BBN does not allow processes involving $X$ or $q$ to induce recoupling. This means that the BBN constraint forces (some of) the various assumptions we made which led to (4.20) and (4.23) to be violated. Note that we made those assumptions so that those processes could induce recoupling. Thus, our analysis shows that if recoupling occurred for $T>\Lambda$, it would be incompatible with BBN. ${ }^{7}$ Therefore, recoupling can occur only for $T<\Lambda$ and the only reaction that can recouple neutrinos is the $\nu_{L} \nu_{L} \rightarrow \bar{\nu}_{R} \bar{\nu}_{R}$ process (and the "bar" of this process), which we have discussed at the beginning of this section.

What kind of CMB signals do we predict? First, a $\Delta N_{\nu}$ signal will not exist. Since $T_{\text {rec }}<\Lambda$, there are no heavy particles which are in equilibrium with neutrinos after recoupling. The confinement phase transition may produce some latent heat, but that occurs at $T \approx \Lambda$ which is before recoupling, and therefore has no consequence. This can be a very useful prediction, because if we see a $\Delta N_{\nu}$ signal, then we know that the theory - if it is a composite neutrino theory - must have chiral symmetry breaking.

In contrast, a $\Delta \ell$ signal should be very robust. As long as the bound (4.14) is satisfied for a mass eigenstate, we always have a $\Delta \ell$ signal from the scattering of any two neutrinos of that eigenstate, because, for instance, the rate of the process, $\nu_{R} \nu_{R} \rightarrow \nu_{R} \nu_{R}$, is larger by $T^{4} / m_{\nu}^{4}$ than the rate of the $\nu_{L} \nu_{L} \rightarrow \bar{\nu}_{R} \bar{\nu}_{R}$, which is already ensured to be faster than the expansion rate by (4.14). Furthermore, since the bound (4.14) depends on $m_{\nu}$, the $\Delta \ell$ signal can probe the neutrino mass spectrum very sensitively! For example, only in the case of the hierarchical spectrum does exists the region where we observe a $\Delta \ell$ signal corresponding to only one neutrino species scattering, i.e., $\Delta \ell \approx 8$. Similarly, if the $\Delta \ell$ signal indicates that two neutrino species are scattering $(\Delta \ell \approx 16)$, then we will know that the spectrum cannot be degenerate. If we see three neutrinos scattering $(\Delta \ell \approx 24)$, then we will have no clue.

\section{Constraints From Terrestrial Experiments}

In section 4.2, we found that in theories without chiral symmetry breaking, CMB signals are present only for a very low confinement scale $\Lambda$ and correspondingly a low messenger mass scale $m_{X}$. So, it is important to check if there are constraints from terrestrial experiments.

\footnotetext{
${ }^{7}$ Depending on how those assumptions are violated, the actual bounds from BBN vary. Here, we simply assume that a conflict with BBN is avoided by adjusting parameters in the "UV theory" that generates the operator (2.1). But, having giving up recoupling, this can be now done easily, because the tension was always between BBN and recoupling.
} 
To discuss terrestrial bounds, we need to specify how the standard model couples to the nu-color sector, because we can certainly go beyond the scale $M$, so the operator (2.1) must be "resolved" in terms of renormalizable operators. Recall the requirement that the communication should not involve any irrelevant operator. Interestingly and fortunately, everything we need for analyzing physics in terrestrial experiments is uniquely fixed. Schematically, it is

$$
\mathcal{L}=\alpha \ell N h+m_{N} N N^{c}+\left(\text { Terms with } N^{c}, q, X\right)
$$

where we have introduced two neutral fermions, $N$ and $N^{c}$. Note that it is necessary to introduce a pair of $N$ and $N^{c}$ for each generation in order to give masses to all three generations. Therefore, both $\alpha$ and $m_{N}$ in (5.1) are $3 \times 3$ matrices. However, after electroweak symmetry breaking, $\langle h\rangle=\left(0, v+h^{0}\right)$, we can always first rotate $N$ and $N^{c}$ to diagonalize $m_{N}$, then rotate $e_{L}, \nu_{L}$ and $N$ to diagonalize the charged lepton mass matrix and $\alpha$, while simultaneously rotating $N^{c}$ in the opposite manner as $N$ such that $m_{N}$ remains diagonal. So, we can assume without loss of generality that both $\alpha$ and $m_{N}$ are diagonal and all the flavor violations are put in $U_{M N S}$, which we will not write explicitly below because we know that matrix elements of $U_{M N S}$ are $\mathcal{O}(1)$.

Therefore, for each generation, we have a $3 \times 3$ mass matrix spanned by $\nu_{L}, N$ and $N^{c}$. This can be "diagonalized" as

$$
\alpha v \nu_{L} N+m_{N} N N^{c}+\text { h.c. }=0 \cdot \nu_{0} \nu_{0}+m_{D} N N^{\prime}+\text { h.c. }
$$

where the mass "eigen" states, $\nu_{0}$ and $N^{\prime}$, are given by

$$
\begin{aligned}
\nu_{L} & =\nu_{0} \cos \theta_{N}+N^{\prime} \sin \theta_{N}, \\
N^{c} & =-\nu_{0} \sin \theta_{N}+N^{\prime} \cos \theta_{N},
\end{aligned}
$$

and the mass, $m_{D}$, and the mixing angle, $\theta_{N}$, by

$$
m_{D}=\sqrt{\alpha^{2} v^{2}+m_{N}^{2}} \quad, \quad \sin \theta_{N}=\frac{\alpha v}{m_{D}} \quad, \quad \cos \theta_{N}=\frac{m_{N}}{m_{D}} .
$$

Therefore, at this point, each generation consists of a massless Weyl fermion $\nu_{0}$ and a massive Dirac fermion $\Psi_{N}$ made of $N$ and $N^{\prime}$. We will not consider a case where $m_{D} \lesssim \mathrm{MeV}$, because the very motivation for considering composite neutrinos is to explain why the Dirac masses of neutrinos are so small compared to those of the charged fermions. Hence, we assume that $m_{D}$ is no smaller than the electron mass.

Now, recall that in the standard cosmology, neutrinos decouple from the rest of the universe at $T \approx \mathrm{MeV}$. In our case, because of the mixing (5.3), $\nu_{0}$ decouples at $T \approx \mathrm{MeV} /\left(\cos \theta_{N}\right)^{4 / 3}$, strictly speaking. However, since $\cos \theta_{N} \simeq 1$ as we will see 
shortly, we just say $\nu_{0}$ decouples at the standard temperature, $T \approx \mathrm{MeV}$. The situation with $N^{\prime}$ is more complicated. First of all, if they decouple while they are relativistic, then the the decoupling temperature is given by $T \approx \mathrm{MeV} /\left(\sin \theta_{N}\right)^{2 / 3}$. However, since $m_{D} \gtrsim \mathrm{MeV}$, they soon become non-relativistic before BBN. Alternatively, they may be already non-relativistic when they decouple. In either case, a $N^{\prime}$ decays rapidly into $\nu_{0}$ 's via $N^{\prime} \rightarrow \nu_{0} \nu_{0} \bar{\nu}_{0}$ with the lifetime of order $\left(m_{\mu} / m_{D}\right)^{5} / \sin ^{2} \theta_{N}$ times the muon lifetime, $10^{-6}$ sec. In order not to screw up BBN, they must decay before $\nu_{0}$ 's decouple, so we impose

$$
\frac{m_{\mu}^{5}}{m_{D}^{5} \sin ^{2} \theta_{N}} \times 10^{-6} \text { sec } \lesssim 10^{-2} \text { sec }
$$

which gives

$$
m_{D} \sin ^{\frac{2}{5}} \theta_{N} \gtrsim 10 \mathrm{MeV}
$$

- Muon decay:

First, note that the mixing angle clearly cannot be too large, because if $\nu_{L}$ is made predominantly of $N^{\prime}$, then (5.6) would be in conflict with the direct upper bound, $m_{\nu}<2.2 \mathrm{eV}$, obtained by combining tritium $\beta$-decay experiments with neutrino oscillation data. We will sharpen this point below.

The most precise measurement of $G_{F}$ comes from muon decay. In our case, if $m_{D}<m_{\mu}$, there are three decay modes: $\mu \rightarrow e \nu_{0} \bar{\nu}_{0}, \mu \rightarrow e \nu_{0} \bar{N}^{\prime}$ and $\mu \rightarrow e N^{\prime} \bar{\nu}_{0}$, with the rates proportional to $\cos ^{4} \theta_{N}, \sin ^{2} \theta_{N}, \sin ^{2} \theta_{N}$, respectively. We neglect the case where both of the final neutrinos are $N^{\prime}$, because $\theta_{N}$ should be small as we argued above. When it decays to a $N^{\prime}$, the rate depends on $m_{D}$ rather than $m_{e}$, because $m_{D}>\mathrm{MeV}>m_{e}$. Therefore, for $m_{D}<m_{\mu}$, the muon decay rate is given by $G_{F}^{2} m_{\mu}^{5} / 192 \pi^{3}$ times

$$
\cos ^{4} \theta_{N}\left(1-\frac{8 m_{e}^{2}}{m_{\mu}^{2}}+\mathcal{O}\left(\frac{m_{e}^{4}}{m_{\mu}^{4}}\right)\right)+2 \sin ^{2} \theta_{N}\left(1-\frac{8 m_{D}^{2}}{m_{\mu}^{2}}+\mathcal{O}\left(\frac{m_{D}^{4}}{m_{\mu}^{4}}\right)\right)
$$

Since $G_{F}$ is currently measured down to 4 decimal places [27], we must impose $\sin \theta_{N}<10^{-2}$. However, combining this with (5.6) requires $m_{D}$ to be at least $100 \mathrm{MeV}$, so this analysis which has assumed $m_{D}<m_{\mu}$ is not valid. Therefore, it must be that $m_{D}>m_{\mu}$ and a muon cannot decay into $N^{\prime}$. Then, the standard muon decay rate simply gets multiplied by $\cos ^{4} \theta_{N}$, and requiring that $1-\cos ^{4} \theta_{N}<10^{-4}$ gives $\theta_{N}<10^{-2}$. Therefore, the bound from the muon decay is summarized as

$$
m_{D}>m_{\mu} \text { and } \theta_{N}<10^{-2}
$$


Next, since $\nu_{L}$ and $N^{c}$ mix, a neutrino from a muon $\beta$-decay can further decay into $q$ and $X$ via interactions indicated by $(\cdots)$ in (5.1). Most of them are harmless because we can choose them to be however small we want. There is one process, however, for which we may not have this freedom. It is the process where one $\nu$ goes to $n q$ 's, which we need to generate the operator (2.1). The rate of this process is given by

$$
\approx \frac{\lambda^{2}}{\left(16 \pi^{2}\right)^{n-1}} \frac{G_{F}^{2} m_{\mu}^{5}}{192 \pi^{3}}
$$

Thus, for the worst case of $n=3$ and $\lambda=\mathcal{O}(1)$, the suppression is $\mathcal{O}\left(10^{-4}\right)$, which is right at the experimental bound. So, the $G_{F}$ measurement from muon decay requires $\lambda$ to be less than $\mathcal{O}(1)$ for $n=3$, and no restriction for higher $n$. This is not constraining our theory.

- Invisible $Z$-width:

The $Z$ decay rate into a $\nu_{0} \overline{\nu_{0}}$ pair now gets multiplied by $\cos ^{4} \theta_{N}$. The $Z$ can also decay into a $\nu_{0}-N^{\prime}$ pair if $m_{D}<m_{Z}$, but this rate is proportional to $\sin ^{2} \theta_{N}$, which is very small thanks to (5.8). Therefore, the modification in the invisible $Z$-width is less than 1 part in $10^{4}$, which is smaller than the experimental error in the direct measurement of the invisible $Z$-width - actually even smaller than the error in the $N_{\nu}$ fit by LEP [27]. So, there is no additional constraint from the $Z$-width.

- Lepton flavor violation in $\mu \rightarrow e \gamma$ :

Note that the diagrams with an internal $\nu_{0}$ vanishes by GIM mechanism. When the internal line is $N^{\prime}$, the leading diagrams which do not vanish by GIM need two insertions of $m_{D}$ in the internal line. This means we need one insertion of the muon mass outside, since this decay is a magnetic dipole transition, which flips the helicity. Therefore, the rate is given by

$$
\Gamma_{\mu \rightarrow e \gamma} \approx \frac{1}{16 \pi}\left(\frac{e\left(g \sin \theta_{N}\right)^{2}}{16 \pi^{2}} \frac{m_{\mu} m_{D}^{2}}{m_{W}^{4}}\right)^{2} m_{\mu}^{3}
$$

We should compare this to the standard muon decay rate:

$$
\Gamma_{\mu \rightarrow e \nu \bar{\nu}} \simeq \frac{G_{F}^{2} m_{\mu}^{5}}{192 \pi^{3}} \simeq \frac{g^{4} m_{\mu}^{5}}{6144 \pi^{3} m_{W}^{4}} .
$$

So,

$$
\frac{\Gamma_{\mu \rightarrow e \gamma}}{\Gamma_{\mu \rightarrow e \nu \bar{\nu}}} \approx 10^{-2} \times\left(\frac{m_{D} \sin \theta_{N}}{m_{W}}\right)^{4} \approx 10^{-12} \times\left(\frac{m_{D} \sin \theta_{N}}{100 \mathrm{MeV}}\right)^{4}
$$


Comparing this with the current strictest experimental bound, $10^{-12}[25,26]$, we obtain an upper bound for $m_{D}$ :

$$
m_{D} \lesssim \frac{100 \mathrm{MeV}}{\sin \theta_{N}}
$$

We see that there is a large allowed region in the $m_{D^{-}} \theta_{N}$ space where all the bounds, (5.6), (5.8) and (5.13), are satisfied.

- Events with missing (transverse) energy:

Note that any event with an $N^{\prime}$ emission from a $W$ or $Z$ looks identical to the similar event with a $\nu_{0}$ emission. The problem is that due to the bound (5.8), the rate of the former process is always less than $10^{-4}$ of that of the latter. If $m_{D}$ is sufficiently large, the rate will be further suppressed by a small phase space. Therefore, it is extremely hard to isolate this type of processes.

Next, consider an event where a $N$ is emitted from a Higgs. Note that the coupling of the Higgs to a $\nu_{0}-N$ pair is given by $\alpha \cos \theta_{N} \simeq m_{D} \sin \theta_{N} / v$ rather than $m_{D} / v$. Therefore, because of the bound (5.13), the Higgs coupling to the $\nu_{0}-N$ pair is weaker than that to a $s-\bar{s}$ pair, so again it will be very difficult to see this kind of events.

\section{Conclusions}

The idea of composite right-handed neutrinos is quite interesting, and it provides a very natural, simple rationale for why Dirac neutrinos can be so light. While it is impossible to test this idea in laboratory experiments, it is possible that there are signals in the CMB spectrum if the compositeness scale is sufficiently low.

In scenarios where the underlying strong dynamics breaks chiral symmetry, we have found that the interactions between the neutrinos and the "pions" from the chiral symmetry breaking are quite significant so that the constraint from BBN requires the compositeness scale to be very high $(\gtrsim \mathrm{GeV})$ compared to the relevant energy scale for the $\mathrm{CMB}$ physics $(T \approx \mathrm{eV})$. Consequently, there are no signs of the neutrino compositeness which can be seen in the CMB - although this scenario does have signals the CMB, they are indistinguishable from the signals in scenarios discussed in Ref. [1] where the small Dirac neutrino masses arise from spontaneous breaking of low-energy flavor symmetry. Therefore, unfortunately, the CMB signals are not useful to probe the neutrino compositeness. However, it is still theoretically quite interesting in the sense that a composite $\nu_{R}$ theory with chiral symmetry breaking can offer an ideal mechanism to stabilize the low VEV present in those flavor breaking scenarios. 
On the other hand, if the underlying strong dynamics does not undergo chiral symmetry breaking, a very robust CMB signal can arise - a large $\Delta \ell$ signal can be observed in the entire allowed region in the parameter space thanks to the large scattering crosssection among composite objects. And the allowed region is very large. This is a great virtue, because the $\Delta \ell$ signal is a very unique and clean signal. We have also found that the $\Delta \ell$ signal is very sensitive to the mass spectrum of neutrinos, providing the exciting possibility of determining the neutrino mass spectrum from CMB observations.

In contrast, a $\Delta N_{\nu}$ signal is predicted to be absent. This is also a striking prediction in the sense that if we observe a $\Delta N_{\nu}$ signal, it will mean that the underlying theoryif it is a composite theory - must have chiral symmetry breaking. In this view, the difference between the two cases - with or without chiral symmetry breaking - is very intriguing. It is quite interesting that we can extract such dynamical information from the sky.

We have also found that in the case without chiral symmetry breaking, the compositeness scale should be very low $(\lesssim \mathrm{MeV})$ in order to have the signal. Therefore, we have discussed possible collider constraints and signatures. Surprisingly, and fortunately or unfortunately, if we take the parameters to be such that there are signals in the CMB, we find no additional constraints on the parameters from terrestrial experiments.

\section{Acknowledgments}

I thank Nima Arkani-Hamed for introducing me to and educating me on composite neutrinos. I also thank Lawrence Hall, Ken Lane, Markus Luty, Danny Marfatia, Aaron Pierce, Veronica Sanz, and Martin Schmaltz for useful conversations. Finally, I thank Zackaria Chacko for pointing out a crucial error and also reading the manuscript with many fruitful comments. This work is supported by the U.S. Department of Energy under contract DE-FG03-91ER-40676.

\section{Appendix: Models With Massless Fermionic Composites}

Here we summarize some relevant results from [28]. We discuss two cases, the one without "pions" and the one with "pions".

\section{(a) A Model Without Chiral Symmetry Breaking}

Consider an $S U(6)$ gauge theory with fermions, $\psi_{a I}$ and $\chi^{a b}=-\chi^{b a}$, where $I$ is a flavor index $(I=1,2)$, while $a, b$ are gauge indices $(a, b=1, \cdots, 6)$. The gauge index corresponds to the fundamental of $S U(6)$ if it is downstairs, or the anti-fundamental if it is upstairs. This theory is asymptotically-free and has the following flavor symmetries: 
- An $S U(2)$ symmetry acting on the two $\psi$ s.

- A $U(1)$ symmetry, under which $\psi$ and $\chi$ have charge 2 and -1 , respectively.

- A $Z_{2}$ symmetry, under which $\psi \rightarrow-\psi$.

- A $Z_{4}$ symmetry, under which $\chi \rightarrow i \chi$.

The principle of complementarity applies to this theory, and one can analyze the theory both in the Higgs picture and in the confinement picture to verify that they give consistent results. For our purpose, we would like to describe the results in the confinement language. At low energies beneath the confinement scale, we have three massless "baryons", $B_{I J}=B_{J I}=\psi_{\{I}\left(\psi_{J\}} \chi\right)$, which is a triplet of the flavor $S U(2)$ and has charge 3 under the flavor $U(1)$. The baryons do not transform under the $Z_{2}$, while they do transform as $B \rightarrow i B$ under the $Z_{4}$. All the flavor symmetries remain unbroken at low energies, and indeed one can check that all of continuous and discrete anomalies match. Since the theory has no flavor symmetry breaking, there are no "pions", and therefore these three baryons are the only massless particles from this theory.

\section{(b) A Model With Chiral Symmetry Breaking}

Consider an $S U(7)$ gauge theory with fermions, $\psi_{a b c}^{I}$ and $\chi_{S}^{a b}$, where $I$ and $S$ are flavor indices $(I=1,2,3$ and $S=1,2)$, while $a, b, c$ are gauge indices $(a, b, c=1, \cdots, 7)$. The gauge index corresponds to the fundamental of $S U(7)$ if it is downstairs, or the anti-fundamental if it is upstairs. All gauge indices are anti-symmetrized. This theory is asymptotically-free and has the following flavor symmetries:

- An $S U(3)$ symmetry acting on the three $\psi$ s.

- An $S U(2)$ symmetry acting on the two $\chi \mathrm{s}$.

- A $U(1)$ symmetry, under which $\psi$ and $\chi$ has charge -1 and 3 , respectively.

- A $Z_{30}$ symmetry, under which $\psi \rightarrow \mathrm{e}^{i \pi / 15} \psi$.

- A $Z_{10}$ symmetry, under which $\chi \rightarrow \mathrm{e}^{i \pi / 5} \chi$.

The principle of complementarity applies to this theory, and one can analyze the theory both in the Higgs picture and in the confinement picture to verify that they give consistent results. For our purpose, we would like to describe the results in the confinement language. At low energies beneath the confinement scale, the flavor symmetries are broken down to the $U(1)$ and $Z_{30}^{\prime} \equiv Z_{30} Z_{10}^{-1}$. We have three massless "baryons", and the 
baryon has charge 7 under the $U(1)$ and transforms as $B \rightarrow \mathrm{e}^{-i 7 \pi / 15} B$ under the $Z_{30}^{\prime}$.

More specifically, $B_{I S}=\epsilon_{I J K} \phi_{a}^{* J} \phi_{b}^{* K} \chi_{S}^{a b}$, where $\phi_{I}^{a}=\epsilon_{I J K} \epsilon^{a b c d e f g} \psi_{b c d}^{J} \psi_{e f g}^{K}$. Although it appears here that there are $3 \times 2=6$ states of $B$ rather than 3 , one can show that 3 of them actually marry with the 3 states of $B^{\prime I}=\epsilon^{J K L} \phi_{J}^{a} \phi_{K}^{b} \phi_{L}^{c} \psi_{a b c}^{I}$ to become massive. Indeed, such masses respect the low-energy flavor symmetries, because $B^{\prime}$ has charge -7 under the $U(1)$ and transforms as $B^{\prime} \rightarrow e^{i 7 \pi / 15} B^{\prime}$ under the $Z_{30}^{\prime}$. The leftover, three states of $B$ remain completely massless. One can verify that all of the anomalies for the $U(1)$ and the $Z_{30}^{\prime}$ match, while the anomalies involving other symmetries do not match. This is in accord with the results from the Higgs picture analysis where there are just three massless fermions with the same quantum number as $B$ and all the flavor symmetries except the $U(1)$ and the $Z_{30}^{\prime}$ are broken by condensates. Therefore, we conclude that the flavor symmetries are partially broken in this theory, and in particular the breaking of $S U(3) \times S U(2) \times U(1)$ down to $U(1)$ produces 11 massless "pions".

\section{References}

[1] Z. Chacko, L. J. Hall, T. Okui and S. J. Oliver, "CMB signals of neutrino mass generation," arXiv:hep-ph/0312267.

[2] N. Arkani-Hamed and Y. Grossman, "Light active and sterile neutrinos from compositeness," Phys. Lett. B 459, 179 (1999) [arXiv:hep-ph/9806223].

[3] Y. Fukuda et al. [Super-Kamiokande Collaboration], "Evidence for oscillation of atmospheric neutrinos," Phys. Rev. Lett. 81, 1562 (1998) [arXiv:hep-ex/9807003];

[4] Q. R. Ahmad et al. [SNO Collaboration], "Direct evidence for neutrino flavor transformation from neutral-current interactions in the Sudbury Neutrino Observatory," Phys. Rev. Lett. 89, 011301 (2002) [arXiv:nucl-ex/0204008].

[5] K. Eguchi et al. [KamLAND Collaboration], "First results from KamLAND: Evidence for reactor anti-neutrino disappearance," Phys. Rev. Lett. 90, 021802 (2003) [arXiv:hep-ex/0212021].

[6] M. B. Smy et al. [Super-Kamiokande Collaboration], "Precise measurement of the solar neutrino day/night and seasonal variation in Super-Kamiokande-I," Phys. Rev. D 69, 011104 (2004) [arXiv:hep-ex/0309011].

[7] T. Gherghetta, "Dirac neutrino masses with Planck scale lepton number violation," Phys. Rev. Lett. 92, 161601 (2004) [arXiv:hep-ph/0312392].

[8] V. Barger, J. P. Kneller, H. S. Lee, D. Marfatia and G. Steigman, "Effective number of neutrinos and baryon asymmetry from BBN and WMAP," Phys. Lett. B 566, 8 (2003) [arXiv:hep-ph/0305075]. 
[9] W. Hu, D. Scott, N. Sugiyama and M. J. White, "The Effect of physical assumptions on the calculation of microwave background anisotropies," Phys. Rev. D 52, 5498 (1995) [arXiv:astro-ph/9505043].

[10] S. Hannestad, JCAP 0305, 004 (2003) [arXiv:astro-ph/0303076].

[11] S. Bashinsky and U. Seljak, "Neutrino Perturbations in CMB Anisotropy and Matter Clustering," arXiv:astro-ph/0310198.

[12] S. Hannestad, JCAP 0502, 011 (2005) [arXiv:astro-ph/0411475].

[13] R. Trotta and A. Melchiorri, arXiv:astro-ph/0412066.

[14] S. Weinberg, "Phenomenological Lagrangians," PhysicaA 96, 327 (1979).

[15] A. Manohar and H. Georgi, "Chiral Quarks And The Nonrelativistic Quark Model," Nucl. Phys. B 234, 189 (1984);

[16] H. Georgi, "Weak Interactions And Modern Particle Theory," (Benjamin/cummings, Menlo Park, USA, 1984);

[17] H. Georgi and L. Randall, "Flavor Conserving CP Violation In Invisible Axion Models," Nucl. Phys. B 276, 241 (1986).

[18] D. N. Spergel et al. "First Year Wilkinson Microwave Anisotropy Probe (WMAP) Observations: Determination of Cosmological Parameters," Astrophys. J. Suppl. 148, 175 (2003) [arXiv:astro-ph/0302209];

[19] M. Tegmark et al. [SDSS Collaboration], "Cosmological parameters from SDSS and WMAP," arXiv:astro-ph/0310723;

[20] S. Hannestad and G. Raffelt, "Cosmological mass limits on neutrinos, axions, and other light particles," arXiv:hep-ph/0312154.

[21] C. Weinheimer et al., "High Precision Measurement Of The Tritium Beta Spectrum Near Its Endpoint And Upper Limit On The Neutrino Mass," Phys. Lett. B 460, 219 (1999);

[22] V. M. Lobashev et al., "Direct Search For Mass Of Neutrino And Anomaly In The Tritium Beta-Spectrum," Phys. Lett. B 460, 227 (1999);

[23] J. Bonn et al., "The Mainz Neutrino Mass Experiment," Nucl. Phys. Proc. Suppl. 91, 273 (2001).

[24] J. F. Beacom, N. F. Bell and S. Dodelson, "Neutrinoless Universe," arXiv:astro-ph/0404585. 
[25] C. Dohmen et al. [SINDRUM II Collaboration.], "Test Of Lepton Flavor Conservation In $\mathrm{Mu} \rightarrow$ E Conversion On Titanium," Phys. Lett. B 317, 631 (1993);

[26] S. Ahmad et al., "Search For Muon - Electron And Muon - Positron Conversion," Phys. Rev. D 38, 2102 (1988).

[27] K. Hagiwara et al. [Particle Data Group Collaboration], "Review Of Particle Physics," Phys. Rev. D 66, 010001 (2002).

[28] S. Dimopoulos, S. Raby and L. Susskind, "Light Composite Fermions," Nucl. Phys. B 173, 208 (1980). 